\title{
On State Accessibility in Reaction Systems
}

\author{
G. Bastin and J. Lévine
}

\begin{abstract}
Reaction systems constitute a class of nonlinear dynamical systems relevant in many engineering fields such as chemical engineering, biotechnology, and ecology. The state accessibility rank of generic reaction systems can be characterized, irrespective of the reaction kinetics, by simple algebraic criteria closely related to the underlying structure of the reaction network and the associated exchange dynamics.
\end{abstract}

\section{INTRODUCTION}

$\mathrm{T}$ HE concept of reaction system refers to a wide class of nonlinear dynamical systems that have been used, for a long time, as useful tools for a better understanding of engineering problems in various fields such as chemical engineering, biomedical engineering, biotechnology, ecology, etc.

The stability of these systems (without control inputs) has been the object of in depth studies in the literature from the early seventies (see e.g., the review article [4]). In contrast, their state accessibility, which is a prerequisite to controllability and is the purpose of this paper, has received much less attention.

The dynamics of reaction systems are commonly described by ordinary nonlinear differential equations (under the form of a state-space model) arising from standard mass-balance considerations. These models result from the combination of a reaction network (which encodes the reactions that are supposed to occur in the system) with two basic physical phenomena: reaction kinetics and exchange dynamics.

As a matter of fact, the inherent structure of both reaction network and exchange dynamics is essentially linear, while the system nonlinearity is concentrated in the reaction kinetics under the form of highly coupled nonlinear rational functions.

Our contribution will be to show that much can be said about the state accessibility of reaction systems independently of the reaction kinetics, by using arguments from linear algebra and linear systems only, in close connection with the underlying structure of the reaction network and

Manuscript received August 5, 1991; revised June 17, 1992. Paper recommended by Past Associate Editor, J. W. Grizzle. This work was supported in part by the Interuniversity Attraction Pole no. 17 of the Belgian Science Policy Programming and in part by a French Ministry of Research and Development Grant.

G. Bastin is with the Centre for Systems Engineering and Applied Mechanics (CESAME), Université Catholique de Louvain, 4-6, Avenue G. Lemaitre, B-1348 Louvain la Neuve, Belgium.

J. Lévine is with the Centre Automatique et Systèmes, Ecole National Supérieure des Mines de Paris, 35, Rue Saint Honoré, F-77305 Fontainebleau, France.

IEEE Log Number 9208454. the exchange dynamics. In particular, we shall describe simple algebraic criteria for the determination of the strong accessibility rank of so-called generic reaction systems without resorting to Lie bracket computations.

Our motivation for examining the state accessibility of reaction systems is threefold.

1) There is a clear industrial interest to be able to characterize the compositions (that is the respective amounts of the various components involved in the reactions) which can be actually achieved in a given application.

2) In many practical cases, one has very little certainty about the reaction kinetics that are really occuring in a system, while the exchange dynamics are much well known. Yet, it is a peculiarity of reaction systems that particular patterns of the exchange dynamics (that are frequent in practice) may produce a lack of full state accessibility. It is therefore of interest to detect beforehand those systems which cannot be full state reachable, irrespective of the knowledge of the kinetics.

3) State accessibility is a necessary requirement for the transfer of persistent excitation needed to the convergence of parameter identification algorithms from input-output data, which is also a key issue in many practical applications concerning reaction systems (e.g., [14], Vajda et al. [3], Chen et al., 1990).

The paper is organized as follows. A detailed presentation of reaction systems is given in the next section, including a description of reaction networks, the main assumptions regarding the reaction kinetics and the exchange dynamics and the derivation of a general statespace model. In Section III, the maximal strong accessibility rank of generic reaction systems is progressively narrowed. A fast procedure for the computation of the maximal strong accessibility rank is presented in Section IV. The stability of the uncontrollable modes is examined in Section V. Some additional properties are finally discussed in Section VI.

\section{DESCRIPTION OF REACTION SYSTEMS}

In this section, we give a formal and self-contained description of reaction systems. We introduce a particular terminology to discuss the state accessibility of these systems. The vocabulary will appear to be closely related to that commonly used in chemical and/or biological engineering, but its scope is actually much larger.

A reaction system takes place inside a closed domain (for instance, a reactor in a chemical engineering application, or a fish-pond in an ecological application). It is characterized by $\boldsymbol{a}$ set of $\boldsymbol{m}$ reactions involving a set of $\boldsymbol{n}$ 
components. Two different mechanisms are supposed to be involved.

- Reaction Kinetics which refer to the transformation of the components inside the domain (for instance, transformation of chemical species through chemical reactions, or growth of a trout population by consumption of nutrients in a pond, or both together.

- Exchange Dynamics which refer to the exchange (without alteration) of the components between the system and its surroundings.

The dynamics of reaction systems are encoded into reaction networks which are now described.

\section{A. Reaction Networks}

We start the description with two simple examples taken from chemical engineering. In each case, the components are designed $X_{1}, X_{2}, \cdots, X_{n}$, numbered in an arbitrary order.

Example 2.1: We consider the so-called Brusselator of Prigogine and Lefever [11] which is described by the following reaction network with $m=3$ reactions involving $n=6$ components:

$$
\begin{gathered}
X_{1} \rightarrow X_{3}+X_{4} \\
X_{2}+X_{3} \rightarrow X_{5}+X_{6} \\
2 X_{3}+X_{5} \rightarrow 3 X_{3} .
\end{gathered}
$$

Example 2.2: A plausible mechanism for the reaction between nitric oxide and hydrogen is described by the following reaction network with $m=4$ reactions and $n=$ 6 components (e.g., [5, ch. 4]):

$$
\begin{gathered}
2 X_{1} \rightarrow X_{3} \\
X_{3} \rightarrow 2 X_{1} \\
X_{2}+X_{3} \rightarrow X_{4}+X_{5} \\
X_{2}+X_{5} \rightarrow 2 X_{6} .
\end{gathered}
$$

The six components are: $X_{1}=\mathrm{NO}, X_{2}=\mathrm{H}_{2}, X_{3}=\mathrm{N}_{2} \mathrm{O}_{2}$, $X_{4}=\mathrm{N}_{2}, X_{5}=\mathrm{H}_{2} \mathrm{O}_{2}, X_{6}=\mathrm{H}_{2} \mathrm{O}$.

Basically, a reaction network is thus a set of $m$ reactions of the following form:

$$
\begin{gathered}
\sum_{i} \gamma_{i j} X_{i} \rightarrow \sum_{i} \delta_{i j} X_{i} \quad j=1, \cdots, m \\
\gamma_{i j} \geq 0 \quad \delta_{i j} \geq 0 \quad \forall i, j .
\end{gathered}
$$

The coefficients $\gamma_{i j}$ and $\delta_{i j}$ are positive real numbers (called stoichiometric coefficients in chemical engineering and yield coefficients in biological engineering). They express the nominal quantity of component $X_{i}$ which is consumed or produced by the $j$ th reaction. For example the third reaction of the network (2.1) means: two moles of $X_{3}$ combined with one mole of $X_{5}$ produces three moles of $X_{3}$.

In Examples 2.1 and 2.2, it appears that all the stoichiometric coefficients are integer numbers, as usual in chemi- cal engineering. In general, however, the coefficients are allowed to be real numbers as illustrated by the following biotechnological example.

Example 2.3: The following reaction network with $m=$ 3 reactions and $n=7$ components is presented by Axelsson [1] on the basis of a paper by Sonnleitner and Käppeli [12], to describe yeast growth on glucose:

$$
\begin{aligned}
X_{1}+2.33 X_{2}+ & 0.525 X_{3} \rightarrow 3.5 X_{4}+2.5 X_{5}+3.66 X_{6} \\
X_{1}+0.054 X_{3} \rightarrow & 0.36 X_{4}+1.89 X_{5}+0.14 X_{6}+1.88 X_{7} \\
1.61 X_{2}+0.198 X_{3} & +X_{7} \\
& \rightarrow 1.32 X_{4}+0.68 X_{5}+2.12 X_{6} .
\end{aligned}
$$

The seven components are: Glucose $X_{1}$, Oxygen $X_{2}$, Ammonia $X_{3}$, Yeast $X_{4}$, Carbon Dioxide $X_{5}$, Water $X_{6}$, Ethanol $X_{7}$.

In the sequel, we shall sometimes consider particular subsets of components. They will be designed by a notation of the form $X^{a}$ where the superscript " $a$ " is an identifier of the subset. $I_{a}$ will denote the associated index, that is the set of the indexes of the components involved in $X^{a}$. Two typical examples are the subsets of reactants and of products.

A reactant is a component which appears on the lefthand side of at least one reaction. The set of reactants is:

$$
\begin{aligned}
& X^{r}=\left\{X_{t} \mid \gamma_{i j}>0 \text { for some } j\right\} \\
& \text { with index } I_{r}=\left\{i \mid \gamma_{i j}>0 \text { for some } j\right\} .
\end{aligned}
$$

Similarly, a product is a component which appears on the right-hand side of at least one reaction. The set of products is:

$X^{p}=\left\{X_{i} \mid \delta_{i j}>0\right.$ for some $\left.j\right\}$

with index $I_{p}=\left\{i \mid \delta_{i j}>0\right.$ for some $\left.j\right\}$.

A component $X_{i}$ can clearly be a reactant of one reaction and a product of the same or another reaction (that is: $\left.I_{r} \cap I_{p} \neq \varnothing\right)$. When a component $X_{i}$ appears on both sides of a reaction $j$, it is called autocatalyst if $\delta_{i j}>\gamma_{i j}>0$ and catalyst if $\delta_{i j}=\gamma_{i j}>0$.

A terminal product is a component which is a product of at least one reaction but a reaction of none of the reactions. The set of terminal products is defined as:

$$
X^{t}=\left\{X_{i} \mid \gamma_{i j}=0 \quad \forall j\right\} \quad \text { index } I_{t}=I_{p} \backslash I_{r}
$$

Similarly, an initial reactant is a component which is a reactant of at least one reaction but a product of none of the reactions. The set of initial reactants is denoted:

$$
X^{d}=\left\{X_{i} \mid \delta_{i j}=0 \forall j\right\} \quad \text { index } I_{d}=I_{r} \backslash I_{p} .
$$


Example 2.4: In the reaction network (2.1) of the Brusselator, we can identify the following subsets of components:

$$
\begin{aligned}
& \text { Reactants: } I_{r}=\{1,2,3,5\} \\
& \text { Products: } I_{p}=\{3,4,5,6\} \\
& \text { Terminal products: } I_{t}=I_{r} \backslash I_{p}=\{4,6\} \\
& \text { Initial reactants: } I_{d}=I_{p} \backslash I_{r}=\{1,2\} \\
& \text { Autocatalyst: } \quad X_{3} \text { in the third reaction. }
\end{aligned}
$$

\section{B. Dynamical State-Space Model}

The dynamical state-space model expresses the material balance of the various components inside the system. It is thus assumed that the presence of each component in the system can be quantized with appropriate units (e.g., molar concentration, units of mass, number of cells, weight of biomass etc.). We denote by $x_{i}(t)$ the (instantaneous) quantity of component $X_{i}$ in the system. The state vector of the model is then the vector of the nonnegative quantities $x_{i}$ :

$$
\text { state vector: } \boldsymbol{x} \equiv\left(x_{1}, x_{2}, \cdots, x_{n}\right)^{T} \text {. }
$$

It is called the composition of the system. The global dynamics of reaction systems result from the combination of two mechanisms: reaction kinetics and exchange dynamics.

Reaction Kinetics: The reaction rates (units of material/unit of time) are the expression of the rate of reactants consumption and products formation in the system, according to the reaction network. A reaction rate $r_{j}(x, t)$ is associated to each reaction of the network $(j=1, \cdots, m)$. It is basically a nonnegative time varying function of the state $x$. In this paper, however, we limit ourselves to the case (fairly common in practice) where the reaction rates do not explicitly depend on time and are rational functions of the states only. The vector of reaction rates (called reaction kinetics) is denoted:

$$
\text { reaction kinetics: } \boldsymbol{r}(\boldsymbol{x}) \equiv\left(r_{1}(\boldsymbol{x}), r_{2}(\boldsymbol{x}), \cdots, r_{m}(\boldsymbol{x})\right)^{T} \text {. }
$$

Each reaction rate $r_{j}(x)$ may be a highly complex nonlinear function of the states $x_{i}$ but with a slight restriction due to the following basic "physical" fact: a reaction can take place only if all the reactants of that reaction are present in the reactor, or, in other words, a reaction rate is necessarily zero whenever the quantity of one of its reactants is zero. This is technically stated in the following assumption.

Assumption 2.1:

1) $r_{j}(x) \geq 0 \forall j$

2) $r_{j}(x)=0$ if $x_{i}=0$ for some $i \in I_{r j}$

where $I_{r j}$ denotes the index of the reactants involved in the $j$ th reaction.
A special case of particular interest is when the reaction rates obey the so-called mass-action principle:

$$
r_{j}(x)=k_{j}\left(\prod_{i \in I_{r j}} x_{i}^{\alpha_{i j}}\right)
$$

where $k_{j}$ denotes the rate constant of the $j$ th reaction. The mass-action principle consists of expressing each reaction rate as being proportional to the product of the involved reactant quantities $x_{i}(t)$, each raised to a power $\alpha_{i j}$ called the order of the $j$ th reaction with respect to the $i$ th component.

Exchange Dynamics: A basic feature of so-called open reaction systems is that some of the reactants are continuously supplied to the system from the outside and that all the components may be continuously removed from the system. The set of supplied reactants is denoted $X^{s}$ (index $\left.I_{s}, \operatorname{dim}\left(I_{s}\right) \equiv q\right)$. In particular, we assume that all the initial reactants belong to $X^{s}$, that is $I_{d} \subseteq I_{s}$. In addition, we adopt the notational convention that the supplied reactants are numbered from 1 to $q$ :

$$
X^{s} \equiv\left\{X_{i}, i=1, \cdots, q\right\} .
$$

The feed rates of the supplied reactants are denoted $u_{i}$ $(i=1, \cdots, q)$.

Moreover, all the components are supposed to be removed from the system at a rate proportional to their quantity $x_{i}$. The proportionality constants are called specific removal rates and are denoted $d_{i}(\geq 0, i=1, \cdots, n)$. When all the components are removed at the same positive specific rate (i.e., $d_{i}=d>0$ for all $i$ ), the outflow is said to be homogeneous. When only some of the $d_{i}$ 's are equal, it is said partially homogeneous.

State-Space Model: On the grounds of our previous definitions, the balance of each component in the system is now readily seen to be written as follows:

$$
\dot{x}_{i}=\sum_{j=1}^{m}\left(\delta_{i j}-\gamma_{i j}\right) r_{j}(x)-d_{i} x_{i}+\left\{\begin{array}{ll}
u_{i} & 1 \leq i \leq q \\
0 & q<i \leq n
\end{array} .\right.
$$

We introduce the following matrix notations:

$$
\begin{aligned}
& \boldsymbol{u}=\left(u_{1}, u_{2}, \cdots, u_{q}\right)^{T} \quad B=\left(\begin{array}{c}
\boldsymbol{I}_{q} \\
\mathbf{0}
\end{array}\right) \\
& \Gamma=\left[\gamma_{i j}\right] \quad n \times m \text { matrix with entries } \gamma_{i j} \\
& \Delta=\left[\delta_{i j}\right] \quad n \times m \text { matrix with entries } \delta_{i j} \\
& \boldsymbol{D}=\operatorname{diag}\left\{d_{i}, i=1, \cdots, n\right\} \text {. }
\end{aligned}
$$

The dynamics of the system are then represented by the following dynamical state-space model:

$$
\dot{x}=(\Delta-\Gamma) r(x)-D x+B u .
$$

We also define the characteristic matrix:

$$
\boldsymbol{C}=\Delta-\Gamma \text {. }
$$

The rank $p$ of this matrix is often called the rank of the reaction network (e.g., [4]). With the definition (2.6), the state-space model is rewritten:

$$
\dot{x}=C r(x)-D x+B u .
$$


Throughout the paper, a dynamical system described by this state-space model will be called a reaction system.

Example 2.5: Consider the Brusselator reaction network (2.1). Assume that the reactants $X_{1}$ and $X_{2}$, which are the only reactants which are not produced in the system, are the supplied reactants. The state-space model is as follows:

$$
\begin{aligned}
\left(\begin{array}{l}
\dot{x}_{1} \\
\dot{x}_{2} \\
\dot{x}_{3} \\
\dot{x}_{4} \\
\dot{x}_{5} \\
\dot{x}_{6}
\end{array}\right)=\left(\begin{array}{ccc}
-1 & 0 & 0 \\
0 & -1 & 0 \\
1 & -1 & 1 \\
1 & 0 & 0 \\
0 & 1 & -1 \\
0 & 1 & 0
\end{array}\right)\left(\begin{array}{l}
r_{1}(\boldsymbol{x}) \\
r_{2}(\boldsymbol{x}) \\
r_{3}(\boldsymbol{x})
\end{array}\right) \\
-\left(\begin{array}{l}
d_{1} x_{1} \\
d_{2} x_{2} \\
d_{3} x_{3} \\
d_{4} x_{4} \\
d_{5} x_{5} \\
d_{6} x_{6}
\end{array}\right)+\left(\begin{array}{ll}
1 & 0 \\
0 & 1 \\
0 & 0 \\
0 & 0 \\
0 & 0 \\
0 & 0
\end{array}\right)\left(\begin{array}{l}
u_{1} \\
u_{2}
\end{array}\right) .
\end{aligned}
$$

The rank of the reaction network in this case is $p=3=$ $m$.

Comment 2.1: It may arise that the rate constants $k_{j}$ that premultiply the rate functions (2.5) are temperature dependent (according to the Arrhenius law for instance). For the moment, we assume that the temperature is regulated at an appropriate value so that the system operates in isothermal conditions and the rate constants are really constant. The nonisothermal case will be addressed in Section VI.

Comment 2.2: It is common practice, in chemical engineering, to deal with reversible reactions depicted by reaction schemes of the following kind:

$$
X_{1} \rightleftharpoons X_{2}+X_{3}
$$

i.e., with two arrows in opposite directions. The consistency of our description of reaction systems requires to encode reversible reactions as two separate simple reactions, one for each direction, as follows:

$$
\begin{aligned}
& X_{1} \rightarrow X_{2}+X_{3} \\
& X_{2}+X_{3} \rightarrow X_{1} .
\end{aligned}
$$

This means that each reversible reaction is actually counted twice in the reaction network. This was for instance the case in Example 2.2 where the first two reactions actually stand for a single reversible reaction.

Example 2.6: Consider the reaction network (2.2) with the two supplied reactants $X_{1}$ (nitric oxid) and $X_{2}$ (hydro- gen). The state-space model is as follows:

$$
\begin{gathered}
\left(\begin{array}{l}
\dot{x}_{1} \\
\dot{x}_{2} \\
\dot{x}_{3} \\
\dot{x}_{4} \\
\dot{x}_{5} \\
\dot{x}_{6}
\end{array}\right)=\left(\begin{array}{cccc}
-2 & 2 & 0 & 0 \\
0 & 0 & -1 & -1 \\
1 & -1 & -1 & 0 \\
0 & 0 & 1 & 0 \\
0 & 0 & 1 & -1 \\
0 & 0 & 0 & 2
\end{array}\right)\left(\begin{array}{l}
r_{1}(\boldsymbol{x}) \\
r_{2}(\boldsymbol{x}) \\
r_{3}(\boldsymbol{x}) \\
r_{4}(\boldsymbol{x})
\end{array}\right) \\
-\left(\begin{array}{l}
d_{1} x_{1} \\
d_{2} x_{2} \\
d_{3} x_{3} \\
d_{4} x_{4} \\
d_{5} x_{5} \\
d_{6} x_{6}
\end{array}\right)+\left(\begin{array}{ll}
1 & 0 \\
0 & 1 \\
0 & 0 \\
0 & 0 \\
0 & 0 \\
0 & 0
\end{array}\right)\left(\begin{array}{l}
u_{1} \\
u_{2}
\end{array}\right) .
\end{gathered}
$$

In this case, due to the presence of a reversible reaction in the scheme, the characteristic matrix has not full rank: the rank of the reaction network is $p=3<m=4$.

Comment 2.3: The notion of reaction system covers a very wide spectrum of application fields. Obviously, the first field one has in mind is chemical engineering since our description coincides with the usual presentation of open isothermal reactors. But the concept can be extended in many other fields such as biotechnology (see, for instance, Example 2.3, and also [2]), ecology, pharmacokinetics, etc.... For example, the famous LotkaVolterra prey-predator model (e.g., [8]), is as follows:

$$
\left(\begin{array}{l}
\dot{x}_{1} \\
\dot{x}_{2}
\end{array}\right)=\left(\begin{array}{cc}
c_{1} & -1 \\
0 & c_{2}
\end{array}\right)\left(\begin{array}{l}
r_{1}(\boldsymbol{x}) \\
r_{2}(\boldsymbol{x})
\end{array}\right)-\left(\begin{array}{ll}
0 & 0 \\
0 & d
\end{array}\right)\left(\begin{array}{l}
x_{1} \\
x_{2}
\end{array}\right)+\left(\begin{array}{l}
1 \\
0
\end{array}\right) u_{1}
$$

with the reaction rates

$$
r_{1}(\boldsymbol{x}) \equiv x_{1} \quad r_{2}(\boldsymbol{x}) \equiv x_{1} x_{2} .
$$

This state-space model is easily seen to correspond to the following reaction network (see also [9, ch. 5]):

$$
\begin{gathered}
X_{1} \rightarrow\left(1+c_{1}\right) X_{1} \\
X_{1}+X_{2} \rightarrow\left(1+c_{2}\right) X_{2} .
\end{gathered}
$$

The component $X_{1}$ represents the preys and the component $X_{2}$ represents the predators. The reaction rate $r_{1}(\boldsymbol{x})=x_{1}$ is the growth rate of the self-reproducing population of preys on an unlimited renewable resource while $r_{2}(x)=x_{1} x_{2}$ represents the growth rate of the predator population by consumption of the preys. The specific removal rate $d$ is the mortality rate of the predators. The feed rate $u_{1}$ represents a supply of preys into the system.

Epidemics of diseases give rise to another class of dynamical models that can be interpreted in terms of reaction networks. For example, in the classical model of Kermac and McKendrick [7] a contagious disease spreads among a population by contact between the individuals. The infected individuals are denoted $X_{1}$. The uninfected but susceptible individuals are denoted $X_{2}$. Progressively, 
the infected individuals become immune $X_{3}$. This situation can be described by the following reaction network:

$$
\begin{gathered}
X_{1}+X_{2} \rightarrow 2 X_{1} \\
X_{1} \rightarrow X_{3} .
\end{gathered}
$$

The first reaction represents contagion while the second represents recovery. The associated state-space model will be derived by completing the description with appropriate exchange dynamics (i.e., migration of the involved populations). Obviously, the reaction network could also be augmented by introducing additional effects such as procreation and vaccination...

\section{StRong ACCESSIBILITY OF REACTION SySTEMS}

The concept of accessibility in reaction systems relies on the possibility, in a given application, of steering the system from one composition to another one. The following definition of accessibility is relevant for reaction systems of the form (2.7), see e.g., [13].

Definition 3.1: A composition $\boldsymbol{x}^{(\mathbf{1})}$ is accessible from a composition $\boldsymbol{x}^{(\mathbf{0})}$ if there exist a finite time $T$ and a feeding function $u(t)$ such that if $x(0)=x^{(0)}$ then $x(T)=$ $\boldsymbol{x}^{(1)}$.

An important question concerning the notion of accessibility is the dimension of the manifold which is strongly accessible at time $T$ from a given composition $\boldsymbol{x}$. This dimension is called the strong accessibility rank at $\boldsymbol{x}$. For the readers who are not familiar with the concept of state accessibility, some basic definitions are recalled in the Appendix.

The strong accessibility rank $\rho$ of a reaction system (2.7) is the dimension of the following Lie algebra:

$$
\rho=\operatorname{dim} \operatorname{Lie}\{\boldsymbol{C r}(\boldsymbol{x})-\boldsymbol{D} \boldsymbol{x} \mid \operatorname{col} \boldsymbol{B}\}
$$

where col $\boldsymbol{B}$ denote the columns of $\boldsymbol{B}$. The aim of this section is to characterize this rank with simple algebraic criteria directly computable from the reaction network without writing the dynamics analytically and without the knowledge of the kinetics. We have the following preliminary fundamental result.

Theorem 3.1: The strong accessibility rank $\rho$ of a reaction system (2.7) satisfies the following inequality:

$$
q \leq \rho \leq \operatorname{rank} \Re
$$

where $\mathfrak{R}$ is the following accessibility matrix:

$$
\mathfrak{A}=\left(\boldsymbol{B}, C, D C, D^{2} C, \cdots, D^{n-1} C\right) .
$$

Proof: The proof proceeds in successive steps.

1) The left inequality is obvious, due to the specific structure of $\boldsymbol{B}$, that is the specific way how the inputs $u_{i}$ enter the system.

2) We have thus to prove that:

$$
\operatorname{dim} \operatorname{Lie}\{\boldsymbol{f}(\boldsymbol{x}) \mid \operatorname{col} \boldsymbol{B}\} \leq \operatorname{dim} \operatorname{sp}\{\operatorname{col} \Re\}
$$

where $f(x)=\boldsymbol{C r}(\boldsymbol{x})-D x$. We shall first prove that inequality (3.1) holds in the single input case: $q=1, \boldsymbol{B}=\boldsymbol{b}$. Clearly, it is sufficient to prove that, if a vector field $\boldsymbol{h}$ belongs to $\operatorname{sp}\{\operatorname{col} \Re\}$, then:

$$
[\boldsymbol{f}, \boldsymbol{h}] \in \operatorname{sp}\{\operatorname{col} \mathfrak{R}\}
$$

since the distribution $\{$ col $\mathfrak{R}\}$ is involutive (As usual [.,.] denotes the Lie brackets operation). The columns of $\Re$ are denoted:

$$
\Re=\left\{c^{(0)}, c^{(1)}, c^{(2)}, \cdots, c^{(m)}, \cdots, c^{(n m)}\right\} .
$$

Since $\boldsymbol{h}$ belongs to $\mathrm{sp}\{\mathrm{col} \mathfrak{\Re}\}$, there exists a set of scalar smooth real valued functions $v_{i}(\boldsymbol{x}), i=0,1, \cdots, n m$, such that:

$$
h(x)=\sum_{i} c^{(i)} v_{i}(x)
$$

3) Proof of (3.2):

$$
\begin{aligned}
{[f, h](x)=\left[f, \sum_{i} c^{(i)} v_{i}\right](x) } \\
=\sum_{i} c^{(i)} \frac{\partial v_{i}(x)}{\partial x} f(x)-C \frac{\partial r}{\partial x} h(x)-\sum_{i} D c^{(i)} v_{i}(x)
\end{aligned}
$$

(I)

(II)

We clearly have:

(I) $\in \operatorname{sp}\{\operatorname{col} \mathfrak{R}\}$,

(II) $\in \operatorname{sp}\{\operatorname{col} C \subseteq \operatorname{sp}\{\operatorname{col} \Re\}$, and

(III) $\in \operatorname{sp}\left\{D b, D C, D^{2} C, \cdots, D^{n} C\right\} \subseteq \operatorname{sp}\{\operatorname{col} \Re\}$

because $D b$ is colinear to $\boldsymbol{b}$, and from Cayley-Hamilton theorem.

4) The recurrence is initiated as follows:

$$
h(x)=[f, b]=-C \frac{\partial r}{\partial x} b+D b \in \operatorname{sp}\{b, \operatorname{col} C\} .
$$

5) The argumentation of steps 2), 3), 4) is readily extended to the multiinput case.

It is worth noting that the computation of $\operatorname{rank}(\mathfrak{A})$ relies only on the knowledge of the characteristic matrix $C$ and the exchange matrix $D$, but does not require the knowledge of the kinetics vector function $r(x)$. Furthermore it follows from the proof of Theorem 3.1 that the sequence of distributions $Q_{j}$ (see the Appendix) satisfy, in the present case, the following set of inclusions:

$$
Q_{j} \subseteq \operatorname{span}\left\{\operatorname{col}\left(B, C, D C, D^{2} C, \cdots, D^{i-1} C\right)\right\} .
$$

In the generic case where all the distributions $Q_{j}$ are nonsingular, we have in addition (according to Lemma A.2, Appendix):

$$
Q_{j} \subseteq \operatorname{span}\left\{\operatorname{col}\left(B, C, D C, D^{2} C, \cdots, D^{n-2} C\right)\right\} \quad \forall j .
$$

This leads to the following corollary of Theorem 3.1.

Corollary 3.1: The maximal strong accessibility rank of a generic reaction system is:

$$
\rho_{m}=\operatorname{rank} \Re_{m}
$$


where $\Re_{m}$ denotes the following maximal accessibility matrix:

$$
\Re_{m}=\left(B, C, D C, D^{2} C, \cdots, D^{n-2} C\right) \text {. }
$$

Note the difference between $\Re$ and $\Re_{m}$ the latter being obtained by dropping the last bloc $D^{n-1} C$ from the first one.

One of the main interests of Corollary 3.1 is to give a simple criterion to detect beforehand, from the structure of the reaction network, those generic reaction systems which are not fully accessible, that is which possess uncontrollable modes, or otherwise stated for which the strong accessibility rank $\rho$ is certainly strictly lesser than the number of components $n$. As a matter of fact, the maximal accessibility rank $\rho_{m}$ is closely related to the structure of the exchange dynamics and more precisely to the outflow homogeneity. We have the following result.

Theorem 3.2: The maximal strong accessibility rank of a generic reaction system satisfies the following inequality:

$$
\operatorname{rank}(\boldsymbol{B}, \boldsymbol{C}) \leq \rho_{m} \leq n .
$$

The upper bound is achieved for heterogeneous flow systems having their specific removal rates all different (i.e., $d_{i} \neq d_{j}$ for all $i, j$ ). The lower bound is reached for homogeneous flow systems where all the components are removed at the same specific rate (i.e., $d_{i}=d$ for all $i$ ). Furthermore, for such systems, if $q+p<n$ (that is number of supplied reactants + rank of the reaction network lesser than number of components), then the system cannot be fully accessible.

Proof: The upper bound is straightforward from the structure of the maximal accessibility matrix (3.3). The lower bound is an immediate consequence of Corollary 3.1 if one observes that $\boldsymbol{D}=d I_{n}$ for homogeneous flow systems. The last statement results $\operatorname{from} \operatorname{rank}(B, C) \leq$ rank $\boldsymbol{B}+\operatorname{rank} \boldsymbol{C}$.

We now illustrate Corollary 3.1 and Theorem 3.2 with a simple example.

Example 3.1: We consider the case of a simple reaction system described by the following single autocatalytic reaction with three components:

$$
X_{1}+X_{2} \rightarrow 2 X_{2}+X_{3} \text {. }
$$

We assume that $X_{1}$ is the single supplied reactant and that the kinetics obey the law of mass action. The system dynamics are written as follows:

$$
\left(\begin{array}{c}
\dot{x}_{1} \\
\dot{x}_{2} \\
\dot{x}_{3}
\end{array}\right)=\left(\begin{array}{c}
-1 \\
1 \\
1
\end{array}\right) x_{1} x_{2}-\left(\begin{array}{l}
d_{1} x_{1} \\
d_{2} x_{2} \\
d_{3} x_{3}
\end{array}\right)+\left(\begin{array}{l}
1 \\
0 \\
0
\end{array}\right) u_{1} .
$$

We calculate the distributions $Q_{j}$ for this system:

$$
\begin{array}{r}
Q_{0}=\operatorname{span}\left\{\left(\begin{array}{l}
1 \\
0 \\
0
\end{array}\right)\right\} \quad Q_{1}=\operatorname{span}\left\{\left(\begin{array}{l}
1 \\
0 \\
0
\end{array}\right),\left(\begin{array}{c}
-1 \\
1 \\
1
\end{array}\right) x_{2}\right\} \\
Q_{2}=\operatorname{span}\left\{\left(\begin{array}{l}
1 \\
0 \\
0
\end{array}\right),\left(\begin{array}{c}
-1 \\
1 \\
1
\end{array}\right) x_{2},\left(\begin{array}{c}
-d_{1} \\
d_{2} \\
d_{3}
\end{array}\right) x_{2}\right\} .
\end{array}
$$

We first observe that this reaction system will be nongeneric only when $x_{2}=0$ which defines clearly a thin set in the state space. We thus assume that $x_{2} \neq 0$ and we calculate $\mathfrak{R}_{m}$ :

$$
\Re_{m}=\left(\begin{array}{ccc}
1 & -1 & -d_{1} \\
0 & 1 & d_{2} \\
0 & 1 & d_{3}
\end{array}\right) .
$$

We observe that

1) the maximal strong accessibility rank is $\rho_{m}=$ rank $\Re_{m}=n=3$ when $d_{2} \neq d_{3}$ and is $\rho_{m}=\operatorname{rank} \Re_{m}=$ $\operatorname{rank}[\boldsymbol{B}, \boldsymbol{C}]=2$ when $d_{2}=d_{3}$, in agreement with Theorem 3.2 and

2) in each case, the actual strong accessibility rank is the maximal one: $\rho=\rho_{m}$.

From this example, it appears clearly that, for generic reaction systems, the maximal strong accessibility rank can be achieved in general $\left(\rho=\rho_{m}\right)$ since additional rank decrease can arise only for singular choices of the kinetics vector function $\boldsymbol{r}(\boldsymbol{x})$. This point will be further discussed in Section VI-A.

\section{A Fast PRocedure to Compute $\rho_{m}$}

From Theorem 3.2, it appears that the maximal strong accessibility rank of a generic system lies somewhere between $\operatorname{rank}[\boldsymbol{B}, \boldsymbol{C}]$ and $n$, provided the outflow is partially homogeneous. The whole information needed for the determination of $\rho_{m}$ is contained in the structure of the characteristic matrix $C$ and more precisely in a set of partial homogeneity conditions (that is some of the $d_{i}$ 's are equal) that produce a decrease of the rank of the maximal accessibility matrix. The following procedure allows an exhaustive listing of these conditions.

Procedure:

Step 1): Consider the $(n-q) \times m$ matrix $C^{*}$ obtained by removing the first $q$ rows of $\boldsymbol{C}$. The index of the rows of $C^{*}$ is denoted:

$$
I^{*}=[(q+1),(q+2), \cdots, n] .
$$

Step 2): Find all index sets $I_{j} \subseteq I^{*}$ such that there exist a zero linear combination of rows of $C^{*}$, with nonzero coefficients of the following form:

$$
\sum_{i \in I_{j}} \lambda_{i j} a^{(i)}=0 \text { with } \lambda_{i j} \neq 0 \forall i \in I_{j}
$$

where $a^{(i)}\left(i \in I^{*}\right)$ denote the rows of $C^{*}$ and $I_{j}$ denotes the index of the nonzero coefficients $\lambda_{i j}$.

Step 3): For each $I_{j}$, define a partial homogeneity condition as follows:

$$
d_{i}=d \forall i \in I_{j} .
$$

Definition 4.1: Two partial homogeneity conditions are independent if their index sets $I_{j 1}$ and $I_{j 2}$ do not fully 
overlap, that is:

$$
I_{j 1} \not \subset I_{j 2} \text { and } I_{j 2} \not \subset I_{j 1} \text {. }
$$

The following theorem, stating the rule for computing the maximal accessibility rank of generic reaction system is an immediate consequence of the above procedure.

Theorem 4.1: The maximal accessibility rank $\rho_{m}$ of a generic reaction system is $\rho_{m}=n-h$, where $h$ is the minimal number of independent partial homogeneity conditions that are satisfied by the system.

Example 4.1: Consider the following reaction network:

$$
\begin{gathered}
X_{1}+X_{2} \rightarrow 2 X_{2}+X_{3}+X_{4} \\
X_{1}+X_{3} \rightarrow 2 X_{5}
\end{gathered}
$$

where $X_{1}$ is the (single) supplied reactant. For this system, the maximal accessibility matrix $\mathfrak{R}_{m}$ is as follows:

$\Re_{m}=\left(\begin{array}{ccccccccc}1 & -1 & -1 & -d_{1} & -d_{1} & -d_{1}^{2} & -d_{1}^{2} & -d_{1}^{3} & -d_{1}^{3} \\ 0 & 1 & 0 & d_{2} & 0 & d_{2}^{2} & 0 & d_{2}^{3} & 0 \\ 0 & 1 & -1 & d_{3} & -d_{3} & d_{3}^{2} & -d_{3}^{2} & d_{3}^{3} & -d_{3}^{3} \\ 0 & 1 & 0 & d_{4} & 0 & d_{4}^{2} & 0 & d_{4}^{3} & 0 \\ 0 & 0 & 2 & 0 & 2 d_{5} & 0 & 2 d_{5}^{2} & 0 & 2 d_{5}^{3}\end{array}\right)$.

According to Theorem $3.2,3 \leq \rho_{m} \leq 5$ in this example. We apply the procedure to compute $\rho_{m}$.

Step 1): The matrix $\boldsymbol{C}^{*}$ is as follows:

$C^{*}=\left(\begin{array}{cc}1 & 0 \\ 1 & -1 \\ 1 & 0 \\ 0 & 2\end{array}\right)$ with the associated index: $I^{*}$

$=[2,3,4,5]$.

Step 2): For this matrix, the following three index subsets are easily identified:

$$
\begin{aligned}
& I_{1}=[2,4] \text { with } \lambda_{21}=1 \text { and } \lambda_{41}=-1 \\
& I_{2}=[2,35] \text { with } \lambda_{22}=-2, \lambda_{32}=2, \lambda_{52}=1 \\
& I_{3}=[3,4,5] \text { with } \lambda_{32}=2, \lambda_{42}=-2, \lambda_{52}=1 .
\end{aligned}
$$

Clearly, $\rho_{m}=\operatorname{rank}\left(\Re_{m}\right)=5$ when all the specific removal rates are different that is $d_{i} \neq d_{j}$ for all $(i, j)$. But, according to Theorem 4.1, the accessibility rank decreases in the following cases:

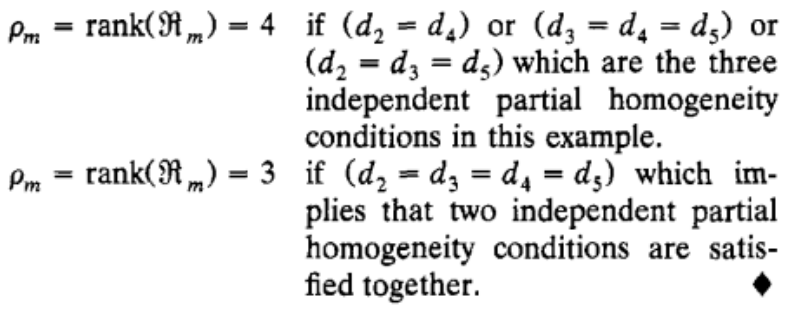

\section{STABILITY OF THE UNCONTROLLABLE MODES}

In this section, we examine the stability of the uncontrollable modes of generic reaction systems that have maximal strong accessibility rank (i.e., $\rho=\rho_{m}$ ) but are not fully strongly accessible (i.e., $\rho<n$ ).
Theorem 5.1: There exists a linear change of coordinates such that, in the new coordinates, the uncontrollable modes of a generic reaction system of maximal strong accessibility rank are governed by linear and stable dynamics.

Proof: It follows from Theorem 4.1 that the number of uncontrollable modes is the minimal number of independent partial homogeneity conditions which are satisfied by the system. We suppose that a set of such conditions has been selected and numbered from 1 to $h$. For each of these conditions, we introduce a new coordinate:

$$
z_{j}=\sum_{i \in I_{j}} \lambda_{i j} x_{i} \quad j=1, \cdots, h
$$

where $\lambda_{i j}$ denote the coefficients defined at the second step of the procedure to compute $\rho_{m}$ described in Section IV. A direct calculation then shows that these new coordinates represent the uncontrollable modes of the system and are governed by the following stable linear dynamics:

$$
\dot{z}_{j}=-d_{j} z_{j} \quad j=1, \cdots, h
$$

where $d_{j}$ denotes the common value of the specific removal rate in the concerned index $I_{j}$.

Example 5.1: The state-space model associated to the reaction network (4.1) is as follows:

$$
\left(\begin{array}{l}
\dot{x}_{1} \\
\dot{x}_{2} \\
\dot{x}_{3} \\
\dot{x}_{4} \\
\dot{x}_{5}
\end{array}\right)=\left(\begin{array}{cc}
-1 & -1 \\
1 & 0 \\
1 & -1 \\
1 & 0 \\
0 & 2
\end{array}\right)\left(\begin{array}{l}
r_{1}(x) \\
r_{2}(x)
\end{array}\right)-\left(\begin{array}{l}
d_{1} x_{1} \\
d_{2} x_{2} \\
d_{3} x_{3} \\
d_{4} x_{4} \\
d_{5} x_{5}
\end{array}\right)+\left(\begin{array}{l}
1 \\
0 \\
0 \\
0 \\
0
\end{array}\right) u_{1} \text {. }
$$

If $d_{2}=d_{4}$, then $\operatorname{rank}\left(\Re_{m}\right)=4$ as we have shown in Example 4.1. There is one uncontrollable mode defined as:

$$
z_{1}=\lambda_{21} x_{2}-\lambda_{41} x_{4}=x_{2}-x_{4} \quad \dot{z}_{1}=-d_{2} z_{1} .
$$

If $d_{2}=d_{3}=d_{4}=d_{5}$, the $\operatorname{rank}\left(\Re_{m}\right)=3$ and there are two uncontrollable modes which may be chosen as:

$$
\begin{aligned}
& z_{1}=x_{2}-x_{4} \quad \dot{z}_{1}=-d_{2} z_{1} \\
& z_{2}=2\left(x_{3}-x_{2}\right)+x_{5} \quad \dot{z}_{2}=-d_{2} z_{2} .
\end{aligned}
$$

\section{ADDITIONAL PROPERTIES}

In this last section, we present some additional properties regarding the accessibility of reaction systems. The discussion is partially inspired by the comments of the reviewers on a first version of the paper.

\section{A. Independent Kinetics and Equivalent Systems}

Without loss of generality, it is always admissible to assume that the kinetics are independent in the sense of the following definition.

Definition 6.1: The kinetics $r_{1}(\boldsymbol{x}), r_{2}(\boldsymbol{x}), \cdots, r_{m}(\boldsymbol{x})$ are independent at the composition $\boldsymbol{x}$ if and only if there exists a neighborhood of $x$ such that the vector space 
$\operatorname{span}\left\{r_{1}(\boldsymbol{x}), r_{2}(\boldsymbol{x}), \cdots, r_{m}(\boldsymbol{x})\right\}$ has dimension $m$ on that neighborhood.

It is easy to see that a reaction system defined with dependent kinetics can always be transformed into an equivalent reaction system with independent kinetics. By equivalent, we mean that the two systems have exactly the same state space representation. Before to give a formal statement of this property, we illustrate the point with two simple examples.

Example 6.1: Consider the following reaction network:

$$
\begin{aligned}
& X_{1} \rightarrow X_{2} \\
& X_{1} \rightarrow X_{3} .
\end{aligned}
$$

We assume that $X_{1}$ is the single supplied reactant and that the kinetics obey the law of mass action:

$$
r_{1}(x)=k_{1} x_{1} \quad r_{2}(x)=k_{2} x_{1} .
$$

These kinetics are proportional to one another and therefore not independent in the sense of Definition 6.1. The state-space model is written as follows:

$$
\left(\begin{array}{l}
\dot{x}_{1} \\
\dot{x}_{2} \\
\dot{x}_{3}
\end{array}\right)=\left(\begin{array}{cc}
-1 & -1 \\
1 & 0 \\
0 & 1
\end{array}\right)\left(\begin{array}{l}
k_{1} x_{1} \\
k_{2} x_{1}
\end{array}\right)-\left(\begin{array}{l}
d_{1} x_{1} \\
d_{2} x_{2} \\
d_{3} x_{3}
\end{array}\right)+\left(\begin{array}{l}
1 \\
0 \\
0
\end{array}\right) u_{1}
$$

with a characteristic matrix

$$
C=\left(\begin{array}{cc}
-1 & -1 \\
1 & 0 \\
0 & 1
\end{array}\right) \quad \operatorname{rank}(C)=2
$$

Now by introducing another reaction rate $r^{o}(x)=x_{1}$ it is easily shown that the model (6.1) is equivalent to

$$
\left(\begin{array}{c}
\dot{x}_{1} \\
\dot{x}_{2} \\
\dot{x}_{3}
\end{array}\right)=\left(\begin{array}{c}
-\left(k_{1}+k_{2}\right) \\
k_{1} \\
k_{2}
\end{array}\right) r^{o}(\boldsymbol{x})-\left(\begin{array}{l}
d_{1} x_{1} \\
d_{2} x_{2} \\
d_{3} x_{3}
\end{array}\right)+\left(\begin{array}{l}
1 \\
0 \\
0
\end{array}\right) u_{1} .
$$

This model itself can be associated with the following reaction network:

$$
\left(k_{1}+k_{2}\right) X_{1} \rightarrow k_{1} X_{2}+k_{2} X_{3}
$$

with a characteristic matrix

$$
C^{o}=\left(\begin{array}{c}
-\left(k_{1}+k_{2}\right) \\
k_{1} \\
k_{2}
\end{array}\right) \quad \operatorname{rank}\left(C^{o}\right)=1 .
$$

Hence, it appears that these two different reaction networks with two different kinetics have the same state space representation because the following equality holds:

$$
\boldsymbol{C r}(\boldsymbol{x})=\boldsymbol{C}^{o} \boldsymbol{r}^{o}(\boldsymbol{x}) \text {. }
$$

We observe also that the number of reactions and the rank of the reaction network are reduced by the transformation (from 2 to 1 ). However, this rank reduction is not systematic as shown by the next example.
Example 6.2: The reaction is defined as:

$$
\begin{aligned}
X_{1}+X_{2} & \rightarrow X_{2}+X_{3} \\
X_{2} & \rightarrow X_{3} \\
X_{2} & \rightarrow X_{1} .
\end{aligned}
$$

Again, we assume that the kinetics obey the law of mass action and are therefore not independent:

$\boldsymbol{r}(\boldsymbol{x})=\left(\begin{array}{c}x_{1} x_{2} \\ x_{2} \\ x_{2}\end{array}\right) \quad \boldsymbol{C}=\left(\begin{array}{ccc}-1 & 0 & 1 \\ 1 & -1 & -1 \\ 0 & 1 & 0\end{array}\right) \quad \operatorname{rank}(C)=2$.

The system is equivalent to a reaction system with independent kinetics defined as:

$$
\boldsymbol{r}^{\circ}(\boldsymbol{x})=\left(\begin{array}{c}
x_{1} x_{2} \\
x_{2}
\end{array}\right) \quad \boldsymbol{C}^{\circ}=\left(\begin{array}{cc}
-1 & 1 \\
1 & -2 \\
0 & 1
\end{array}\right) \quad \operatorname{rank}\left(\boldsymbol{C}^{o}\right)=2
$$

In this example, the number of reactions is reduced from 3 to 2 , but the rank of the reaction network is not reduced and remains equal to 2 .

Property 6.1: Consider a reaction system with characteristic matrix $\boldsymbol{C}$ and kinetics $\boldsymbol{r}(\boldsymbol{x})$ that satisfy Assumption 2.1 and are not independent. Then, there exists an equivalent reaction system with characteristic matrix $\boldsymbol{C}^{\circ}$ and kinetics $\boldsymbol{r}^{\circ}(\boldsymbol{x})$ such that:

1) $\boldsymbol{C r}(\boldsymbol{x})=\boldsymbol{C}^{\circ} \boldsymbol{r}^{\circ}(\boldsymbol{x})$

2) rank $C^{o} \leq \operatorname{rank} C$

3) the kinetics $\boldsymbol{r}^{\circ}(\boldsymbol{x})$ are independent and satisfy Assumption 2.1.

The proof of this property is easy. The way is indicated by the above examples.

The implication of this property is that the matrix $C$ can be replaced by the matrix $\boldsymbol{C}^{\circ}$ in all the previous results. This remark is however of marginal interest because the situations where something is to be gained with this substitution and where the reduction does not appear trivially (as in the above examples) are very seldom (in fact, we were not able to find a realistic one!).

\section{B. Continuous Stirred Tank Reactors}

The continuous stirred tank reactors (CSTR) constitute a very important special class of reaction systems. They are characterized by homogeneous outflow patterns. This means that all the components are withdrawn at the same positive specific rate: $d_{i}=d>0$ for all $i$, with $d$ called dilution rate. For such systems, the foregoing theory provides very simple algebraic tests to detect those continuous stirred tank reactors that are certainly not controllable by manipulating the reactant feeding rates, whatever the form of the kinetics. (Controllability means that any final composition is accessible from any initial one, in a finite time).

Property 6.2: Two simple necessary conditions for a continuous stirred tank reactor to be controllable are:

$$
\operatorname{rank}(\boldsymbol{B}, \boldsymbol{C})=n \quad \text { and } \quad q+\operatorname{rank}(\boldsymbol{C}) \geq n .
$$

This property is a trivial consequence of Theorem 3.2. 
Nonisothermal Reactors: So far, as mentioned in Comment 2.1, we have assumed that the kinetics $r(x)$ depend only on the state $\boldsymbol{x}$ but are not affected by other factors such as the temperature for instance. For many continuous stirred tank chemical reactors, it is however clear that a perfect isothermal operation is the exception rather than the rule. The foregoing accessibility theory can fortunately be extended to nonisothermal reactors in a very straightforward way. Let us assume that the kinetics are function of the composition $\boldsymbol{x}$ and of the temperature $T$ : $r(x, T)$. The dynamics of the system are then obtained by augmenting the previous model (2.7) with an additional energy balance equation (with appropriate normalizations) as follows:

$$
\begin{gathered}
\dot{T}=\sum_{j=1}^{m} h_{j} r_{j}(\boldsymbol{x}, T)-d T+u_{0} \\
\dot{\boldsymbol{x}}=\boldsymbol{C r}(\boldsymbol{x}, T)-\boldsymbol{D} \boldsymbol{x}+\boldsymbol{B u}
\end{gathered}
$$

where the constant coefficients $h_{j}$ represent the heat of reactions $\left(h_{j}>0\right.$ for exothermic reactions, $h_{j}<0$ for endothermic ones) while $u_{0}$ represents the heating/cooling action. It appears that this extended model has exactly the same form (2.7) as before, provided the notations are augmented as:

$$
\begin{aligned}
& \boldsymbol{x} \rightarrow\left(\begin{array}{c}
T \\
\boldsymbol{x}
\end{array}\right) \quad \boldsymbol{C} \rightarrow\left(\begin{array}{c}
h_{1}, \cdots, h_{m} \\
\boldsymbol{C}
\end{array}\right) \\
& \boldsymbol{r}(\boldsymbol{x}) \rightarrow \boldsymbol{r}(\boldsymbol{x}, T) \quad u \rightarrow\left(\begin{array}{c}
u_{0} \\
\boldsymbol{u}
\end{array}\right) .
\end{aligned}
$$

It follows that all the accessibility results we have presented in this paper are directly extendable to the nonisothermal case.

\section{Reaction Invariants and Uncontrollable Modes}

It is interesting to notice that the dynamics (5.1) of the uncontrollable modes also define invariant sets in the space of the original coordinates:

$$
\begin{gathered}
z_{j}(0)=\sum_{i \in I_{j}} \lambda_{i j} x_{i}(0)=0 \Rightarrow z_{j}(t)=\sum_{i \in I_{j}} \lambda_{i j} x_{i}(t)=0 \\
\forall t \text { and } \forall j=1, \cdots, h .
\end{gathered}
$$

These invariant sets associated to the uncontrollable modes form a subset of the set of the so-called reaction invariants that are classically considered for closed reaction systems in the Chemical Engineering literature (see e.g., [15], [16]). The number of invariants in a closed reaction system is $n-\operatorname{rank}(\boldsymbol{C})$, whatever the form of the kinetics. The analysis of this paper can be interpreted as a way of determining the maximal number of invariants that can be dropped out in a given reaction system by manipulating the reactant feeding rates. In fact, we have shown that in a generic system with maximal accessibility rank, the number of remaining invariants (which is the number of uncontrollable modes) is $n-\operatorname{rank}\left(\Re_{m}\right) \leq n-$ $\operatorname{rank}(\boldsymbol{B}, \boldsymbol{C})$. Obviously, when the system is full state accessible, all the invariants disappear.

\section{CONCLUSIONS}

Simple algebraic criteria have been presented in this paper for the direct computation of the strong accessibility rank of reaction systems, from the knowledge of the reaction network structure only. Furthermore, it has been shown that, in case where the system is generically not full state accessible, the uncontrollable modes are necessarily governed by stable linear dynamics, in appropriate coordinates.

\section{ACKNOWLEDGMENT}

The authors would like to thank reviewer 4 for suggestions and comments that contributed to a significant improvement of the paper. They would also like to thank V. Vermeulen for his help in preparing the manuscript of this paper.

\section{APPENDIX \\ STATE ACCEsSibility of NonLINEAR SYSTEMS, A BRIEF REVIEW}

We consider the class of nonlinear systems of the form:

$$
\dot{\boldsymbol{x}}=f(x)+\sum_{i=1}^{p} u_{i} g_{i}(x)
$$

with inputs $u_{i} \in R(i=1, p)$, state $x \in R^{n}$ and where $f(\cdot), g_{i}(\cdot)$ are $n$-dimensional smooth functions on $R^{n}$.

We assume that, in a neighborhood $U$ of a point $\boldsymbol{x}^{\circ}$ in $R^{n}$, there exists a change of coordinates

$$
\left(\begin{array}{l}
w_{1} \\
w_{2}
\end{array}\right)=\phi(x)
$$

such that in the new coordinates, the system dynamics are written as:

$$
\begin{aligned}
& \dot{\boldsymbol{w}}_{1}=f_{1}\left(\boldsymbol{w}_{1}, \boldsymbol{w}_{2}, u_{1}, \cdots, u_{p}\right) \\
& \dot{\boldsymbol{w}}_{2}=f_{2}\left(\boldsymbol{w}_{2}\right) .
\end{aligned}
$$

The coordinates $\boldsymbol{w}_{2}$ are called uncontrollable modes at $\boldsymbol{x}^{\circ}$ because their behavior is clearly not affected by the control inputs $u_{i}$. By extension, the subsystem $w_{2}=f_{2}\left(w_{2}\right)$ may be called the uncontrollable subsystem at $x^{o}$.

It is obviously natural to consider the largest uncontrollable subsystem whose dimension is denoted $\gamma$. The dimension $\rho=n-\gamma$ of the complementary subsystem is then called the strong accessibility rank from $\boldsymbol{x}^{o}$ : it is clearly the dimension of the largest subsystem whose behavior is affected by the control inputs $u_{i}$. It is also the dimension of the manifold accessible from $x^{\circ}$ by manipulating the control inputs $u_{i}$.

Lemma A.1: (e.g., [10, ch. 3]). The strong accessibility rank of a nonlinear system of the form (A1) is the dimension of the Lie algebra that contains the set of vector fields $\boldsymbol{G}=\operatorname{span}\left\{\boldsymbol{g}_{i}(i=1, p)\right\}$, the set of vector fields:

$$
\boldsymbol{F}=\left\{a d_{f}^{k} \boldsymbol{g}_{i},(i=1, p),(k=1, \infty)\right\}
$$


and all the repeated Lie brackets of vector fields from the sets $\boldsymbol{F}$ and $\boldsymbol{G}$. This Lie algebra is denoted:

$$
\operatorname{Lie}\left\{f, \mid g_{1}, g_{2}, \cdots, g_{p}\right\} \text {. }
$$

In practice, the following algorithm can be used to compute the strong accessibility rank (e.g., [6, ch. 1]). The following sequence of distributions is introduced:

$$
\begin{aligned}
& Q_{0}=\operatorname{span}\left\{\boldsymbol{g}_{i},(i=1, \boldsymbol{p})\right\} \\
& Q_{j}=Q_{j-1}+\left[\boldsymbol{f}, Q_{j-1}\right]+\sum_{i=1, \boldsymbol{p}}\left[\boldsymbol{g}_{i}, Q_{j-1}\right] .
\end{aligned}
$$

Lemma A.2: The strong accessibility rank is the dimension of the smallest distribution $Q_{j^{*}}$ such that $Q_{j^{*}}=Q_{j^{*}+1}$. In the particular generic case where all the distributions of the sequence are nonsingular, the number of steps $j^{*}$ is necessarily finite and lesser than $n: j^{*}<n$.

\section{REFERENCES}

[1] J. P. Axelsson, "Modeling and control of fermentation processes," Ph.D. dissertation, Rep. CODEN: LUTFD2/(TFRT-1030)/1-112, 1989, Dept. Automat. Contr., Lund Inst. of Tech., Sweden.

[2] G. Bastin and D. Dochain, On-Line Estimation and Adaptive Control of Bioreactors. Amsterdam: Elsevier, 1990.

[3] L. Chen, G. Bastin, and D. Dochain, "Structural identifiability of yield parameters in nonlinear biological models," in Proc. 29th IEEE Conf. Decision Contr., Honolulu, Dec. 1990.

[4] M. Feinberg, "Chemical reaction network structure and the stability of complex isothermal reactors," Rev. Art. 25, Chemical Engineering Science, vol. 42, no. 10, pp. 2229-2268, 1987.

[5] C. G. Hill, An Introduction to Chemical Engineering Kinetics and Reactor Design. New York: Wiley, 1977.

[6] A. Isidori, Nonlinear Control Systems, 2nd ed. New York: Springer-Verlag, 1989.

[7] W. O. Kermack and A. G. McKendrick, "Contribution to the mathematical theory of epidemics," in Proc. Roy. Soc. A 115, pp. 700-721, 1927.

[8] A. J. Lotka, "Undamped oscillations derived from the law of mass action," J. Amer. Chem. Soc., vol. 42, pp. 1595-1599, 1920.

[9] J. D. Murray, Mathematical Biology, 2nd ed. New York: Springer-Verlag, 1990

[10] H. Nijmeijer and A. van der Schaft, Nonlinear Dynamical Control Systems. New York: Springer-Verlag, 1990.
[11] I. Prigogine and R. Lefever, "Symmetry breaking instabilities in dissipative systems," J. Chem. Phys., vol. 48, pp. 1665-1700, 1968.

[12] B. Sonnleitner and O. Käppeli, "Growth of Sacchamomyces cerevisiae is controlled by its limited respiratory capacity: Formulation and verification of a hypothesis," Biotechnology and Bioengineering, vol. 28, pp. 927-937, 1986.

[13] H. J. Sussmann, "Existence and uniqueness of minimal realizations of nonlinear systems," Math. Syst. Theory, vol. 10, pp. 263-284, 1977.

[14] S. Vajda, H. Rabitz, E. Walter, and Y. Lecourtier, "Qualitative and quantitative identifiability analysis of nonlinear chemical ki. netic models," Chem. Eng. Comm., vol. 83, pp. 191-219, 1989.

[15] K. V. Waller and P. M. Mäkllä, "Chemical reaction invariants and variants and their use in reactor modeling, simulation and control," Ind. Eng. Chem. Process. Des. Dev., vol. 20, pp. 1-11, 1981.

[16] M. Fjeld, O. A. Asbjørnsen, and K. J. Astrom, "Reaction invariants and their importance in the analysis of eigenvectors, state observability, and controllability of the continuous stirred tank reactor," Chemical Eng. Sci., vol. 29, pp. 1917-1926, 1974.

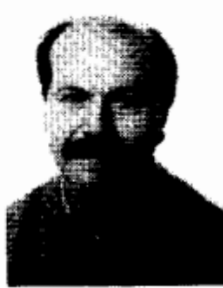

Georges Bastin received the electrical engineering degree and the Ph.D. degree, both from Université Catholique de Louvain, Louvain-laNeuve, Belgium.

$\mathrm{He}$ is currently Professor in the Center for Systems Engineering and Applied Mechanics (CESAME) at the Université Catholique de Louvain. His main research interests are in system identification, nonlinear control theory, adaptive systems and random fields with applications to mechanical systems and robotics, biological processes, transportation systems and environmental problems.

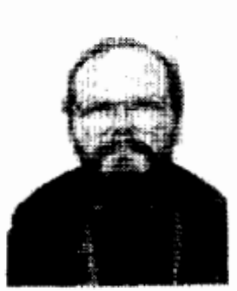

Jean Lévine was born in 1950 in Paris, France. He obtained his Doctorate de 3ème cycle and his Doctorat d'Etat at the University of ParisDauphine in Applied Mathematics in 1976, 1984, respectively.

$\mathrm{He}$ is a Senior Researcher at the "Centre Automatique et Systèmes" (Center for Automatic Control and System Theory) of École des Mines de Paris. His research interests include nonlinear control theory, nonlinear filtering and observers, and their applications to aeronautics, mechanical systems, and chemical processes. 